\title{
Fumonisins and Related Fusarium Species in Pre-harvest Maize Ear Rot in Poland
}

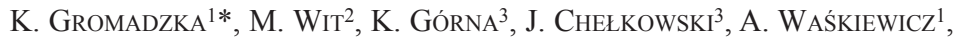 \\ P. OCHODZKI ${ }^{4}$ and R. WARZECHA ${ }^{4}$ \\ ${ }^{1}$ Department of Chemistry, Poznań University of Life Sciences, ul. Wojska Polskiego 75, \\ 60-625 Poznań, Poland \\ ${ }^{2}$ Department of Plant Pathology, Warsaw University of Life Sciences SGGW, Nowoursynowska 159, \\ 00-789 Warsaw, Poland \\ ${ }^{3}$ Institute of Plant Genetics, Polish Academy of Sciences, ul. Strzeszyńska 34, 60-479 Poznań, Poland \\ ${ }^{4}$ Plant Breeding and Accimatization Institute, 05-870 Radzików, Poland
}

(Received 29 April 2016; Accepted 12 August 2016)

Two Fusarium species were identified in mouldy maize ears with the highest frequency during 2005-2014 in 7 seasons: F. subglutinans (3.1-42.0\%) and F. verticillioides (44.1$70.3 \%$ ). Two other species were also found but with lower frequency: $F$. graminearum (1.0-13.0\%) and F. poae (1-45.7\%). In 2005 fumonisin $\mathrm{FB}_{1}$, and in 2013 and 2014 three fumonisins ( $\mathrm{FBs})-\mathrm{FB}_{1}, \mathrm{FB}_{2}$ and $\mathrm{FB}_{3}$ - were identified in harvest samples. The Fusariumdamaged kernel (FDK) fraction contained almost the totality of mycotoxins (90.0-95.0\%), while healthy looking kernels (HLK) contained only below 5.0 to $10.0 \%$. Kernels naturally infected by $F$. verticillioides and $F$. proliferatum contained (in $\mathrm{mg} \mathrm{kg}^{-1}$ ) up to 710.00 of fumonisin $\mathrm{B}_{1}$, up to 209.72 of fumonisin $\mathrm{B}_{2}$ and up to 35.72 of fumonisin $\mathrm{B}_{3}$.

Keywords: mycotoxins, maize ear rot, Fusarium species, fumonisins

\section{Introduction}

Maize (Zea mays L.) is a crop widely grown around the world. Among the main concerns are: maize kernel rot, ear rot, seedling blight, stalk rot, reduction in crop yield worldwide and also grain contamination with mycotoxins (Abbas et al. 2006; Arino et al. 2007). Fusarium species are characterized by exceptional intraspecies and interspecies variability with respect to morphological, physiological, toxigenic and genetic properties (Tancic et al. 2012). An important part of an effective crop protection strategy is monitoring the Fusarium species associated with maize as well as with the specific environment. Temperature may be one factor that determines the extent of invasion of the stalk and ear by Fusarium spp. by affecting both plant and fungal growth. Fusarium verticillioides is more common in regions with hot growing conditions, especially before or during pollination (Pascale et al. 2002; Murillo-Williams and Munkvold 2008). The most important secondary metabolites produced by $F$. verticillioides include group B fumonisins (fumonisin $\left.\mathrm{B}_{1}, \mathrm{~B}_{2}, \mathrm{~B}_{3}\right)$.

*Corresponding author; E-mail: kgromadz@up.poznan.pl 
Discovery of fumonisins in 1988 contributed to finding significance of fumonisin $\mathrm{B}_{1}$ and derivatives as frequent contaminants of maize grain. Fumonisin $\mathrm{B}_{1}$ was discovered in F. verticillioides cultures and caused field outbreaks of leukoencephalomalacia in horses and porcine pulmonary oedema in swine, and was found to be hepatotoxic and hepatocarcinogenic to rats (Bezuidenhout et al. 1988; Gelderblom et al. 1988).

The fumonisin content in kernels depends on the origin of isolates, on susceptibility of the host plant and the geographic region (Czembor et al. 2015). Fumonisin production in maize in Poland was restricted to two species, $F$. verticillioides and $F$. proliferatum (Waśkiewicz et al. 2010; Stępień et al. 2011). In commercially available, processed corn products for human consumption (ground corn grain, corn meal, grits, polenta, semolina, cornflakes and sweet corn) contamination with these toxins typically does not exceed $1000 \mathrm{mg} \mathrm{kg}^{-1}$, although in some countries it is higher.

Undesirable effects of FBs in animal and human organisms result from the similar chemical structure of fumonisin $\mathrm{B}_{1}$ compared to sphingosine and sphinganine - substrates of ceramide synthetase, being a key enzyme in the biosynthesis of sphingolipids. $\mathrm{FB}_{1}$ as a specific inhibitor of this enzyme also inhibits the formation of sphingolipids, leading to a reduction of their contents in eukaryotic cells as well as in serum, kidneys, the liver and urine (Direito et al. 2009). Inhibition of sphingosine biosynthesis and increase in sphinganine concentration is the most sensitive indicator of exposure to fumonisins. Disruption of sphingolipid metabolism probably also explains the important biological effects caused by these toxins, e.g. disorders in the cell cycle, an increase in oxidative stress, and cell apoptosis followed by necrosis (Domijan et al. 2008). Based on numerous data concerning contamination of agricultural products with fumonisins and the diseases they cause, the International Agency for Research on Cancer (IARC) in 2002 classified fumonisin B $_{1}$ among substances probably carcinogenic to humans (class 2B) (IARC 2002). Moreover, in 2007 the Regulation of the EC Commission no. 1126/2007 updated the highest admissible concentrations for the two most important fumonisins, $\mathrm{B}_{1}$ and $\mathrm{B}_{2}$, found in corn and its processed products (European Commission Regulation EC 2007).

The aim of the study was to examine the level of fumonisin accumulation in maize kernel samples with pre-harvest maize ear rot in Poland collected in locations within the most intensive maize growing regions and related Fusarium species in seasons between 2005 and 2014.

\section{Materials and Methods}

\section{Fungi isolation and identification}

Maize ear samples (total number 1001) were collected in October of 2005-2009 and 2013-2014 in three locations of the most intensive maize growing fields in Central East, Central West and Southern West Poland. Ears with significant ear rot were scored for Fusarium ear rot rating (1-100\% kernels mouldy, discoloured and shrunken) and placed in separate paper bags, transported to the laboratory and dried at room temperature. Then to identify Fusarium species, surface mycelium and a small piece of kernel from each ear 
were placed on agar plates in duplicate with low nutrient SNA medium (Nirenberg 1981; Kwaśna et al. 1991). After preliminary identification conidia from each culture were transferred both to potato dextrose agar and synthetic SNA low nutrient agar. The identification of Fusarium species was made according to Nelson et al. (1983), Kwaśna et al. (1991) and Leslie and Summerell (2006) manuals.

Kernels of ears were manually separated into two fractions: Fusarium-damaged kernels (FDK) and healthy looking kernels (HLK - symptomless kernels). Then kernels of the FDK fraction of samples colonized by the species $F$. verticillioides and $F$. proliferatum were subjected to chemical analysis of mycotoxins using methodologies described below.

\section{Molecular analyses: DNA extraction, primers and PCR conditions}

To confirm the morphological identification of the Fusarium strains isolated from maize, genomic DNA extraction was done using a CTAB-based method (Mulé et al. 2004; Stępień et al. 2011). A partial sequence of the tefl-alpha gene was amplified using the Ef728M/Tef1R primer combination according to our previous paper (Błaszczyk et al. 2005; Stępień et al. 2011).

\section{DNA sequencing, analysis and comparison to NCBI GenBank sequences}

PCR-amplified DNA fragments for sequence analysis were purified with exonuclease I [Epicentre, Madison, WI, USA] and shrimp alkaline phosphatase [Promega, Madison, WI, USA] using the following program: $30 \mathrm{~min}$ at $37^{\circ} \mathrm{C}$, followed by $15 \mathrm{~min}$ at $80{ }^{\circ} \mathrm{C}$. Both strands were labelled using the BigDyeTerminator 3.1 kit [Applied Biosystems, Foster City, CA, USA], according to Błaszczyk et al. (2005) and the manufacturer's instructions. To remove the remains of the reagents, labelled fragments were precipitated with ethanol. Sequence reading was performed using Applied Biosystems equipment.

Sequences obtained were compared to the NCBI GenBank-deposited sequences to confirm the correct morphological species identification using the BLASTn algorithm (MEGABLAST). Our own collection of Fusarium strains originating from different host species was included for comparative analysis.

\section{Mycotoxin analyses}

The content of fumonisin $\mathrm{B}_{1}$ was examined in corn kernels in 2005, and three fumonisins $\left(B_{1}, B_{2}\right.$ and $\left.B_{3}\right)$ were identified in maize ear samples in seasons 2013-2014.

\section{Chemicals and reagents}

Standards: Fumonisin $\mathrm{B}_{1}, \mathrm{~B}_{2}$ and $\mathrm{B}_{3}$ were provided by Sigma-Aldrich (Steinheim, Germany).

Solvents: Acetonitrile and methanol (HPLC grade) were purchased from Sigma-Aldrich (Steinheim, Germany). 
Reagents: Glacial acetic acid, 2-mercaptoethanol, $o$-phthaldialdehyde, $o$-phosphoric acid, sodium tetraborate, sodium dihydrophosphate, dipotassium phosphate, potassium chloride and paper filter (Whatman 4) were provided by Sigma-Aldrich (Steinheim, Germany). Water (HPLC grade) was obtained from MilliQ system (Millipore, Billerica, MA, USA).

\section{Sample preparation, extraction and HPLC analysis}

The procedure of extraction and purification of FBs was reported in detail previously (Waśkiewicz et al. 2012). Purified FBs were quantitatively determined by the HPLC/FLD method. The fumonisin $\mathrm{B}_{1}, \mathrm{~B}_{2}$, and $\mathrm{B}_{3}$ standard $(5 \mu \mathrm{l})$ or extracts $(20 \mu \mathrm{l})$ were derivatized with 20 or $80 \mu$ of the $o$-phthaldialdehyde (OPA) reagent. After $3 \mathrm{~min}$, the reaction mixture $(10 \mu \mathrm{l})$ was injected onto an HPLC column. Methanol sodium dihydrogen phosphate $(0.1 \mathrm{M}$ in water) solution $(77: 23, \mathrm{v} / \mathrm{v})$ adjusted to $\mathrm{pH} 3.35$ with $o$-phosphoric acid, after filtration through a $0.45 \mu \mathrm{m}$ Waters HV membrane, was used as the mobile phase with a flow rate of $0.6 \mathrm{ml} \mathrm{min} \mathrm{m}^{-1}$. A Waters 2695 apparatus (Waters Division of Millipore, Milford, MA, USA), with a C-18 Nova Pak column $(3.9 \times 150 \mathrm{~mm})$ and a Waters 2475 fluorescence detector $\left(\lambda_{\mathrm{Ex}}=335 \mathrm{~nm}\right.$ and $\left.\lambda_{\mathrm{Em}}=440 \mathrm{~nm}\right)$ were used in the metabolite quantitative determination. The limits of detection (LOD) were $1.0 \mathrm{ng} \mathrm{g}^{-1}$ for $\mathrm{FB}_{1}, \mathrm{FB}_{2}$, and $\mathrm{FB}_{3}$. The limit of quantification (LOQ) was calculated as three times the LOD. Positive results (on the basis of retention time) were confirmed by HPLC analysis and compared with the relevant calibration curve (correlation coefficients for $\mathrm{FB}_{1}, \mathrm{FB}_{2}$, and $\mathrm{FB}_{3}$ were 0.9967 , 0.9983 , and 0.9966 , respectively). The high correlation coefficients $\left(\mathrm{R}^{2}\right)$ of the calibration curves show good linearity of the method in the range of 0-100 $\mathrm{ng} \mathrm{g}^{-1}$. Samples in which the content of fumonisin went beyond the curve were diluted. Recoveries for $\mathrm{FB}_{1}, \mathrm{FB}_{2}$, and $\mathrm{FB}_{3}$ were 93,96 , and $87 \%$ respectively, which were measured in triplicate by extracting the mycotoxins from blank samples spiked with $1.0-100 \mathrm{ng} \mathrm{g}^{-1}$ of the compound. The relative standard deviations were less than $8 \%$.

\section{Statistical analysis}

Arithmetic means and medians of mycotoxin concentrations as well as the frequency of toxin occurrence and infection level (percentage of infected kernels per sample) were calculated using Microsoft Excel.

Recovery rates were estimated in triplicate by extracting mycotoxins from blank samples spiked with 1.0-00 ng g $\mathrm{g}^{-1}$ of the compounds. The limits of detection (LOD) corresponded to the concentration that gave a signal-to-noise ratio of $3: 1$. Precision of the method was evaluated as the relative standard deviation (RSD) of replicate $(n=6)$ measurements of blank samples spiked with mycotoxins at $10 \mathrm{ng} \mathrm{g}^{-1}$. 


\section{Results}

Fusarium species infecting pre-harvest maize ears

Two species were identified as dominating in pre-harvest Fusarium-infected maize ear samples examined in the 2005-2014 season: F. subglutinans and $F$. verticillioides, representing $3.1-43.1 \%$ and $44.1-70.3 \%$, respectively, of the total number of isolates. It is shown in Table 1 that prevailing species in harvest seasons of 2005-2014 varied over the years, depending on agroecological conditions. F. subglutinans frequency was high in seasons 2005, 2007 and 2009, while $F$. verticillioides was found at a higher frequency in all seasons of studies. Frequency of the species $F$. graminearum was $1-13 \%$ and of $F$. poae $1-45.7 \%$. The species $F$. proliferatum was identified only in two seasons (2006, 2014), with frequency of about $2 \%$. Apart from the five species mentioned above, the following species were identified: F. avenaceum, F. culmorum, F. sporitrichioides and species not listed in Table 1 with low frequency: F. tricinctum and F. equiseti.

Identification of 24 Fusarium verticillioides isolates was confirmed using diagnostic sequences of the teflalpha gene and species-specific primers. Sequences of the teflalpha gene were compared with the GenBank nucleotide sequence database and showed 99$100 \%$ homology to type cultures sequences. Both molecular methods gave the same results and differentiated isolates belonging to the closely related species $F$. proliferatum, $F$. subglutinans and $F$. verticillioides as well as other Fusarium species.

Comparing the two years 2013 and 2014, there was found a decrease in the percentage of FDK fraction but a slight increase of the frequency of Fusarium verticillioides occur-

Table 1. Fusarium species isolated from maize with ear rot or kernel rot symptoms

in seven seasons between 2005-2014 in Poland

\begin{tabular}{|c|c|c|c|c|c|c|c|c|}
\hline & \multicolumn{9}{|c|}{ Percentage of Fusarium species isolates } \\
\cline { 2 - 10 } & F. aven & F. prolif & F. cul & F. gram & F. poae & F. sub & F. spor & F. vert \\
\hline 2005 & 0 & 0 & 3.0 & 1.0 & 1.0 & 42.0 & 1.0 & 46.0 \\
\hline 2006 & 0.3 & 2.5 & 0.3 & 0.3 & 0 & 19.0 & 0 & 68.0 \\
\hline 2007 & 0 & 0 & 0 & 7.8 & 0 & 43.1 & 0 & 46.6 \\
\hline 2008 & 0 & 0 & 0 & 4.5 & 0 & 15.3 & 0 & 70.3 \\
\hline 2009 & 0.9 & 0 & 0.9 & 10.3 & 0 & 30.2 & 0 & 53.5 \\
\hline 2013 & 0 & 0 & 0 & 7.1 & 45.7 & 3.1 & 0 & 44.1 \\
\hline 2014 & 0 & 2.0 & 0 & 13.2 & 14.0 & 26.3 & 0 & 46.5 \\
\hline
\end{tabular}

F. aven - Fusarium avenaceum (Fries) Saccardo

F. prolif-Fusarium proliferatum (Matsushima) Nirenberg

F. cul-Fusarium culmorum (W.G. Smith) Saccardo

F. gram-Fusarium graminearum Schwabe

F. poae-Fusarium poae (Peck) Wollenw.

F. sub-Fusarium subglutinans (Wollenw. \& Reinking) Nelson, Toussoun \& Marasas

F. spor-Fusarium sporotrichioides Sherbakoff

F. vert-Fusarium verticillioides (Saccardo) Nirenberg (= F. moniliforme Sheldon) 
rence. Such a significant decrease in the fumonisins content in 2014 could also be associated with the co-occurrence of the other Fusarium species $F$. graminearum and F. poae. In $38.2 \%$ of the samples collected in 2013 there were isolated at least two Fusarium species, out of which in $23.8 \%$ there was $F$. graminearum. In the next year of studies presence of two Fusarium species was found in $46.4 \%$ of total samples, and Fusarium graminearum participation increased to $34.5 \%$. The change of Fusarium species may be related to the decrease in fumonisin content and a significant increase of content of other toxins: zearalenone, deoxynivalenol, nivalenol, moniliformin and beauvericin (paper in preparation).

\section{Mycotoxins accumulated in maize ears}

Amounts of Fusarium mycotoxins in FDK and HLK fractions in previous seasons indicated that the FDK fraction accounted for almost the totality (up to $>95 \%$ ) of fumonisins in the isolate inoculated by $F$. verticillioides (or F. proliferatum) as well as in naturally infected ears. This fraction of kernels was visibly mouldy and covered with mycelium (Pascale et al. 1999 and 2002). The HLK fraction in our previous studies contained very low amounts of mycotoxins. Therefore, the authors decided that mycotoxin analysis would be carried out only in the fraction with disease symptoms (FDK).

The average content of the FDK fraction of maize kernels in different years (20052014) was in the range from $24.81 \%$ in 2009 to $43.81 \%$ in 2013 . In spite of climate

Table 2. Fumonisins content in pre-harvest maize ear rot in Poland, colonized by $F$. verticilliodes and $F$. proliferatum

\begin{tabular}{|c|c|c|c|c|}
\hline Year & $\begin{array}{c}\text { Positive } \\
\text { samples [\%] }\end{array}$ & Min & Max & Average \\
\hline \multicolumn{5}{|c|}{$\mathrm{FB}_{1}\left[\mathrm{mg} \mathrm{kg}^{-1}\right]$} \\
\hline 2005 & 100.0 & 0.150 & 710.00 & 119.25 \\
\hline 2013 & 61.8 & 0.045 & 525.92 & 99.19 \\
\hline 2014 & 98.2 & 0.008 & 103.21 & 3.19 \\
\hline \multicolumn{5}{|c|}{$\mathrm{FB}_{2}\left[\mathrm{mg} \mathrm{kg}^{-1}\right]$} \\
\hline 2005 & - & - & - & - \\
\hline 2013 & 58.2 & 0.003 & 209.72 & 25.85 \\
\hline 2014 & 53.6 & 0.002 & 31.85 & 1.33 \\
\hline \multicolumn{5}{|c|}{$\mathrm{FB}_{3}\left[\mathrm{mg} \mathrm{kg}^{-1}\right]$} \\
\hline 2005 & - & - & - & - \\
\hline 2013 & 41.8 & 0.004 & 35.72 & 7.07 \\
\hline 2014 & 17.9 & 0.002 & 7.24 & 1.10 \\
\hline
\end{tabular}


changes and the use of different maize varieties over the years, the average percentage of fractions with disease symptoms was rather constant. However, an increase of samples with the maximum content of FDK fraction from $77.6(2005)$ to $100 \%(2013,2014)$ was found in several samples.

The percentage of FDK fraction of the total mass of grains does not always indicate the presence of high amounts of mycotoxins. For example, a sample with $F$. verticillioides as the dominating species contained very high amounts of $\mathrm{FB}_{1}\left(525.92 \mathrm{mg} \mathrm{kg}{ }^{-1}\right), \mathrm{FB}_{2}$ $\left(209.72 \mathrm{mg} \mathrm{kg}^{-1}\right)$ and $\mathrm{FB}_{3}\left(37.54 \mathrm{mg} \mathrm{kg}^{-1}\right)$, but only $6.6 \%$ of kernels were found in the FDK fraction.

In 2005 the fumonisin $B_{1}$ content reached an average of 119.25 and a maximum of 710 $\mathrm{mg} \mathrm{kg}^{-1}$ (Table 2). Maize samples examined in the 2013 season contained: fumonisins $\mathrm{B}_{1}$, $\mathrm{B}_{2}$ and $\mathrm{B}_{3}$ up to $525.92,209.72$ and $37.54 \mathrm{mg} \mathrm{kg}^{-1}$, respectively. Samples examined in 2014 contained significantly lower amounts of fumonisins $B_{1}$ with a maximum at 103.21 $\mathrm{mg} \mathrm{kg}^{-1}$, and it was difficult to explain those results (Table 2).

\section{Discussion}

The frequency of $F$. verticillioides from the 1995 season was significantly higher in most years and this species replaced $F$. subglutinans, whose frequency decreased, in particular in the 2006 and 2008 seasons. The same shift of both species was also found in other countries of central Europe (Adler et al. 2002). Ears of the 2013 and 2014 harvest were colonized frequently ( $>38 \%$ and $46 \%$ of samples, respectively) with two species: $F$. poae $+F$. verticillioides or $F$. verticillioides $+F$. graminearum. Maize ear colonization by the two mentioned Fusarium species was significantly influenced by insect damage caused by such pests as the European corn borer (ECB) Ostrinia nubilalis (Lew et al. 1991; Munkvold et al. 1997). We did not find the European corn borer in collected and examined ears before the 2014 harvest. On the other hand, in 2014 a high percentage of ear samples exhibiting kernel rot were injured by Ostrinia nubilalis larvae (paper in preparation). Aspergillus flavus in examined samples of maize ears was not found.

It should be emphasized that $F$. verticillioides may be a primary causal agent of disease, a secondary invader or an endophyte and systemically colonizes kernels. The fungus infects the emerging maize seedlings, the maturing plant and the new kernel. This species was also frequently recovered from healthy maize seeds (Pamphile and Azevedo 2002). Another endophyte of maize, Acremonium zeae, was found to be a producer of antibiotics inhibitory to F. verticillioides and Aspergillus flavus (Wicklow et al. 2005). Interaction of $F$. verticillioides species with maize plant and with other fungi including pathogens $F$. graminearum and $F$. poae, with endophytes such as Acremonium zeae and hyperparasites such as Trichoderma, is very complex and may influence final contamination of kernels with fumonisins.

Recently we have focused attention on interaction of some Trichoderma species with toxigenic Fusarium species including $F$. verticillioides. T. harzianum was able to reduce growth of $F$. verticillioides and production of mycotoxins as well (paper in preparation). Trichoderma atroviride with high inhibitory activity against various Fusarium species 
was isolated from maize ears from the 2005 harvest and with high frequency in 2014 . Isolates of these species were able to significantly reduce growth and mycotoxin production of highly pathogenic Fusarium species (Gromadzka et al. 2009; Jeleń et al. 2013).

Fumonisins identified in maize samples represent well-recognized mycotoxins with proven toxicological and economic importance. Contamination of food commodities by fumonisins has become a serious food safety problem throughout the world. Consumers and farmers are more and more aware that the fumonisins constitute a real threat to human and animal health. In Italy, higher levels of fumonisin $B_{1}$ were often found $(250.0 \mathrm{mg}$ $\mathrm{kg}^{-1}$ ) than those reported for other European countries (Bottalico 1998). Maize kernel samples infected by $F$. verticillioides examined in the second decade of experiments (seasons 1992-1993) accumulated fumonisins $B_{1}$ and $B_{2}$ in amounts up to $273.2 \mathrm{mg} \mathrm{kg}^{-1}$ and $102.6 \mathrm{mg} \mathrm{kg}^{-1}$, respectively (Chełkowski et al. 1994). In Croatia fumonisin $\mathrm{B}_{1}$ and $\mathrm{B}_{2}$ occurred with the highest and mean concentrations of positive samples $\left(\mathrm{FB}_{1}+\mathrm{FB}_{2}\right)$ at 11.66 and $0.65 \mathrm{mg} \mathrm{kg}^{-1}$, respectively (Jurjevic et al. 1999). According to Demir et al. (2010), in Turkey fumonisin $B_{1}$ contamination ranged from 0.05 to 25.72 and $B_{2}$ from 0.05 to $5.7 \mathrm{mg} \mathrm{kg}^{-1}$. In our studies $\mathrm{FB}_{1}$ in the FDK fraction was detected at a much higher concentration of up to $710.0 \mathrm{mg} \mathrm{kg}^{-1}$ (Table 2). In this maize sample the percentage of the fraction with disease symptoms was very low (1.2\%). However, during the studies also maize ears were completely colonized by the fungus (FDK 100\%) at simultaneously a high content of toxins $\left(\mathrm{FB}_{1}-305.17 \mathrm{mg} \mathrm{kg}^{-1}\right)$. Czembor et al. (2015) examined the fumonisin $\mathrm{B}_{1}$ occurrence during 2011-2012 seasons in Poland. All maize kernel samples contained $\mathrm{FB}_{1}$, with average fumonisin $\mathrm{B}_{1}$ contamination ranged from 0.005 to $1.19 \mathrm{mg} \mathrm{kg}^{-1}$.

The problem of fumonisin-contaminated cereals is particularly important in Africa, where maize is the human staple food and is consumed without any processing. In Eastern and Southern Africa fumonisin $\mathrm{B}_{1}$ was detected at concentrations ranging from 0.002 to $1.91 \mathrm{mg} \mathrm{kg}^{-1}$ (92.5\% positive samples), while the sum of fumonisin $\left(\mathrm{B}_{1}+\mathrm{B}_{2}+\mathrm{B}_{3}\right)$ concentrations in the same samples ranged from 0.002 to $2.73 \mathrm{mg} \mathrm{kg}^{-1}$. The results of frequent fumonisin occurrence at a high level combined with very high maize intake indicated that $\mathrm{FB}_{\mathrm{s}}$ levels in maize from Africa regularly exceed the tolerable daily intake for fumonisins (Waalwijk et al. 2008). These are alarming quantities in particular taking into account the maximum recommended levels of this toxin according to the guidance values. The legislation in Europe (EC 2007) set a limit for fumonisins $B_{1}+B_{2}$ of $1 \mathrm{mg} \mathrm{kg}^{-1}$ in maize intended for direct human consumption, and some samples the in literature exceed this limit.

There are promising results on lower accumulation of fumonisins in grains of transgenic Bt maize hybrids than in commercial varieties. Currently available commercial maize hybrids express Cry proteins in kernels and demonstrated low susceptibility to feeding by ECB. The use of Bt hybrids has been widely accepted in the United States but still has encountered opposition in Europe. Transgenic maize hybrids expressing Cry IA(b) protein in kernels can experience less Fusarium infection and insect feeding. Fumonisin accumulation in Bt hybrids was consistently lower than in non-transgenic hybrids (Munkvold et al. 1997, 1999; Ostry et al. 2010). Wu et al. (2004) estimated that Bt 
corn saves farmers in the United States about $\$ 17$ million annually through reduced fumonisin and deoxynivalenol damage alone. The benefits in mycotoxin reduction could be more significant in developing countries, particularly in regions where corn is a staple in the human diet.

The results showed that $F$. verticillioides was highly pathogenic to maize ears under field conditions. This species may contribute to significant contamination of maize with at least three toxic metabolites (fumonisins $\mathrm{B}_{1}, \mathrm{~B}_{2}$ and $\mathrm{B}_{3}$ ).

Prevention of Fusarium mycotoxin formation by proper agronomical practices before and after maize seeding to avoid grain contamination is very important. As Fusarium mycotoxins are produced within the growing crop, it is important to understand how agricultural practices affect final mycotoxin contamination of grain. Such information could then be used to recommend guidelines on good agricultural practice (GAP) to minimize the mycotoxin contamination of maize products.

\section{Acknowledgement}

This research was financially supported from the Polish National Science Centre project 2014/15/B/NZ9/02169.

\section{References}

Abbas, H.K., Cartwright, R.D., Xie, W., Shier, W.T. 2006. Aflatoxin and fumonisin contamination of corn (maize, Zea mays) hybrids in Arkansas. Crop Protection 25:1-9.

Adler, A., Lew, H., Brunner, S., Oberforster, M., Hinterholzer, J., Kulling-Gradinger, C.M., Mach, R.L., Kubiczek, C.P. 2002. Fusaria in Austrian cereals - change in species and toxins spectrum. J. Appl. Genet. 43A:11-16.

Arino, A., Juan, T., Estopanan, G., Gonzalez-Cabo, J.F. 2007. Natural occurrence of Fusarium species, fumonisin production by toxigenic strains, and concentrations of fumonisins B1 and B2 in conventional and organic maize grown in Spain. J. Food Protect. 70:151-156.

Bezuidenhout, S.C., Gelderblom, W.C.A., Gorst-Allman, C.P., Horak, R.M., Marasas, W.F.O., Spiteller, G., Vleggaar, R. 1988. Structure elucidation of the fumonisins, mycotoxins from Fusarium moniliforme. J. Chem. Soc. Chem. Commun. 11:743-745.

Błaszczyk, L., Tyrka, M., Chełkowski, J. 2005. PstIAFLP based markers for leaf rust resistance genes in common wheat. J. Appl. Genet. 46:357-364.

Bottalico, A. 1998. Fusarium diseases of cereals: species complex and related mycotoxins profiles in Europe. J. Plant Pathol. 80:85-103.

Chełkowski, J., Prończuk, M., Visconti, A., Doko, B. 1994. Fumonisins B1 and B2 accumulation in maize kernels inoculated under field conditions with Fusarium moniliforme Sheldon and in naturally infected cobs. Genetica Polonica 35B:333-338.

Czembor, E., Stępień, Ł., Waśkiewicz, A. 2015. Effect of environmental factors on Fusarium species and associated mycotoxins in maize grain grown in Poland. PLoS ONE 10: e0133644.

Demir, C., Simsek, O., Arici, M. 2010. Incidence of Fusarium verticillioides and levels of fumonisin B1 and B2 in corn in Turkey. Food Sci. Biotechnol. 19:1103-1106.

Direito, G.M., Almeida, A.P., Aquino, S., dos Reis, T.A., Pozzi, C.R., Corrêa, B. 2009. Evaluation of sphingolipids in Wistar rats treated to prolonged and single oral doses of fumonisin B1. Int. J. Mol. Sci. 10:50-61.

Domijan, A., Zeljezic, D., Peraica, M., Kovacevic, G., Gregorovic, G., Krstanac, Z., Horvatin, K., Kalafatic, M. 2008. Early toxic effects of fumonisin $B_{1}$ in rat liver. Hum. Exp. Toxicol. 27:895-900. 
European Commission Regulation (EC), 2007. 20071126/EC (28 September 2007). Official J. Eur. Union L255:14-17.

Gelderblom, W.C.A., Jaskiewicz, K., Marasas, W.F.O., Thiel, P.G., Horak, R.M., Vleggaar, R., Kriek, N.P.J. 1988. Fumonisins - Novel mycotoxins with cancer-promoting activity produced by Fusarium moniliforme. Appl. Environ. Microbiol. 54:1806-1811.

Gromadzka, K., Chełkowski, J., Popiel, D., Kachlicki, P., Kostecki, M., Goliński, P. 2009. Solid substrate bioassay to evaluate the effect of Trichoderma and Clonostachys on the production of zearalenone by Fusarium species. WMJ 2:45-52.

International Agency for Research on Cancer (IARC) 2002. Some traditional herbal medicines, some mycotoxins, naphthalene and styrene. IARC Monogr. 82:301-366.

Jeleń, H., Błaszczyk, L., Chełkowski, J., Rogowicz, K., Strakowska, J. 2013. Formation of 6-n-pentyl-2Hpyran-2-one (6-PAP) and Rother volatiles by different Trichoderma species. Mycological Progress 13:589600.

Jurjevic, Z., Solfrizzo, M., Cvjetkovic, B., Avantaggiato, G., Visconti, A. 1999. Ochratoxin A and fumonisins (B1 and B2) in maize from Balkan nephropathy endemic and non-endemic areas of Croatia. Mycotoxin Res. 15:67-80.

Kwaśna, H., Chełkowski, J., Zajkowski, P. 1991. Fusarium (Sierpik), Vol. XXII: Mycota. Institute of Botany, Polish Academy of Sciences. Warszawa, Kraków, Poland.

Leslie, J.F., Summerell, B.A. 2006. The Fusarium Laboratory Manual. Blackwell Publishing. Ames, IA, USA. $388 \mathrm{p}$.

Lew, H., Adler, A.A., Edinger, W. 1991. Moniliformin and European corn borer (Ostrinia nubilalis). Mycotoxin Res. 7A:71-76.

Mulè, G., Susca, A., Stea, G., Moretti, A. 2004. A species-specific PCR assay based on the calmodulin partial gene for identification of Fusarium verticillioides, F. proliferatum and F. subglutinans. Eur. J. Plant Pathol. 110:495-502.

Munkvold, G.P., Hellmich, R.L., Showers, W.B. 1997. Reduced fusarium ear rot and symptomless infection in kernels of maize genetically engineered for European corn borer resistance. Phytopathol. 87:1071-1077.

Munkvold, G.P., Hellmich, R.L., Rice, L.G. 1999. Comparison of fumonisin concentrations in kernels of transgenic Bt maize hybrids and non-transgenic hybrids. Plant Dis. 83:130-138.

Murillo-Williams, A., Munkvold, G.P. 2008. Systemic infection by Fusarium verticillioides in maize plants grown under three temperature regimes. Plant Dis. 92:1695-1700.

Nelson, P.E., Toussoun, T.A., Marasas, W.F.O. 1983. Fusarium species: An Illustrated Manual for Identification. London: Pennsylvania State University Press. University Park, PA, USA. 193 p.

Nirenberg, H.I. 1981. A simplified method for identifying Fusarium spp. occurring on wheat. Can. J. Bot. 59:1599-1609.

Ostry, V., Ovesna, J., Skarkova, J., Pouchova, V., Ruprich, J. 2010. A review on comparative data concerning Fusarium mycotoxins in Bt maize and non-Bt isogenic maize. Mycotoxin Res. 26:141-145.

Pamphile, J.A., Azevedo, J.L. 2002. Molecular characterization of endophytic strains of Fusarium verticillioides (Fusarium moniliforme) from maize (Zea mays L). World J. Microbiol. Biotechnol. 18:391-396.

Pascale, M., Visconti, A., Avantoggiato, A., Prończuk, M., Chełkowski, J. 1999. Mycotoxin contamination of maize hybrids after infection with Fusarium proliferatum. J. of the Science of Food and Agric. 79:20942098.

Pascale, M., Visconti, A., Chełkowski, J. 2002. Ear rot susceptibility and mycotoxin contamination of maize hybrids inoculated with Fusarium species under field conditions. Eur. J. Plant Pathol. 108:645-651.

Stępień, Ł., Koczyk, G., Waśkiewicz, A. 2011. FUM cluster divergence in fumonisins-producing Fusarium species. Fungal Biol. 115:112-123.

Tancic, S., Slavica Stankovic, S., Levic, J., Krnjaja, V., Vukojevic, J. 2012. Diversity of the Fusarium verticillioides and $F$. proliferatum isolates according to their fumonisin $\mathrm{B}_{1}$ production potential and origin. Genetika 44:163-176.

Waalwijk, C., Koch, S., Ncube, E., Allwood, J., Flett, B.C., de Vries, I., Khema, G.H.J. 2008. Quantitative detection of Fusarium spp. and its correlation with fumonisin content in maize from South African subsistence farmers. WMJ 1:37-45. 
Waśkiewicz, A., Goliński, P., Karolewski, Z., Irzykowska, L., Bocianowski, J., Kostecki, M., Weber, Z. 2010. Formation of fumonisins and other secondary metabolites by Fusarium oxysporum and F. proliferatum - a comparative study. Food Add. Contam. 27:608-615.

Waśkiewicz, A., Wit, M., Goliński, P., Chełkowski, J., Warzecha, R., Ochodzki, P., Wakuliński, W. 2012. Kinetics of fumonisin B1 formation in maize ears inoculated with Fusarium verticillioides. Food Add. Contam. 29:1752-1761.

Wicklow, D.T., Roth, S., Deyrup, S.T., Gloer, J.B. 2005. A protective endophyte of maize: Acremonium zeae antibiotics inhibitory to Aspergillus flavus and Fusarium verticillioides. Mycological Res. 109:610-618.

Wu, F., Miller, J.D., Casman, E.A. 2004. The economic impact of Bt Corn resulting from mycotoxin reduction. Toxin Reviews 23:397-424. 